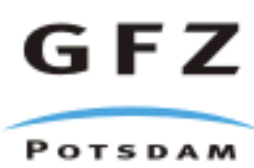

Originally published as:

Steigenberger, P., Rothacher, M., Fritsche, M., Rülke, A., Dietrich, R. (2008): Quality of reprocessed GPS satellite orbits. - Journal of Geodesy, 83, 3-4, 241-248

DOI: 10.1007/s00190-008-0228-7 


\title{
Quality of Reprocessed GPS Satellite Orbits
}

\author{
Peter Steigenberger · Markus Rothacher . \\ Mathias Fritsche · Axel Rülke · Reinhard Dietrich
}

\begin{abstract}
High-precision Global Positioning System (GPS) satellite orbits are one of the core products of the International GNSS Service (IGS). Since the establishment of the IGS in 1994, the quality and consistency of the IGS orbits has steadily been improved by advances in the modeling of GPS observations. However, due to these model improvements and reference frame changes, the time series of operational orbits are inhomogeneous and inconsistent. This problem can only be overcome by a complete reprocessing starting with the raw observation data. The quality of reprocessed GPS satellite orbits for the time period 1994-2005 will be assessed in this paper. Orbit fits show that the internal consistency of the orbits could be improved by a factor of about two in the early years. Comparisons with the operational IGS orbits show clear discontinuities whenever the reference frame was changed by the IGS. The independent validation with Satellite Laser Ranging (SLR) residuals shows an improvement of up to $30 \%$ whereas a systematic bias of $5 \mathrm{~cm}$ still persists.
\end{abstract}

Keywords Global Positioning System · Satellite Orbits · Orbit Modeling · Reprocessing

PACS 91.10.Fc $\cdot 91.10 . \mathrm{Sp}$

\section{Introduction}

One major motivation for the establishment of the International GNSS Service (IGS, Dow et al 2005) on 1 January 1994 was the improvement of the orbit quality. At the beginning of the 1990ies, the accuracy of the GPS orbits was worse than $0.5 \mathrm{~m}$ (Beutler 1993). Starting with GPS week 723 (November 1993), the analysis center (AC) orbit submissions are combined by the IGS analysis center coordinator to form the final IGS orbits. The RMS of the individual AC orbits w.r.t. the combined orbit has improved from about $20 \mathrm{~cm}$ in 1994 to less than $5 \mathrm{~cm}$ in 2005 (Fig. 1). The majority of the AC submissions even agree within $2 \mathrm{~cm}$ with the combined orbit nowadays (Gendt and Kouba 2008). This improvement could only be achieved by a number of model and processing improvements regularly implemented by the ACs. On the other hand, the long-time series of operational IGS orbits are inconsistent due to these processing changes. In particular the quality and consistency

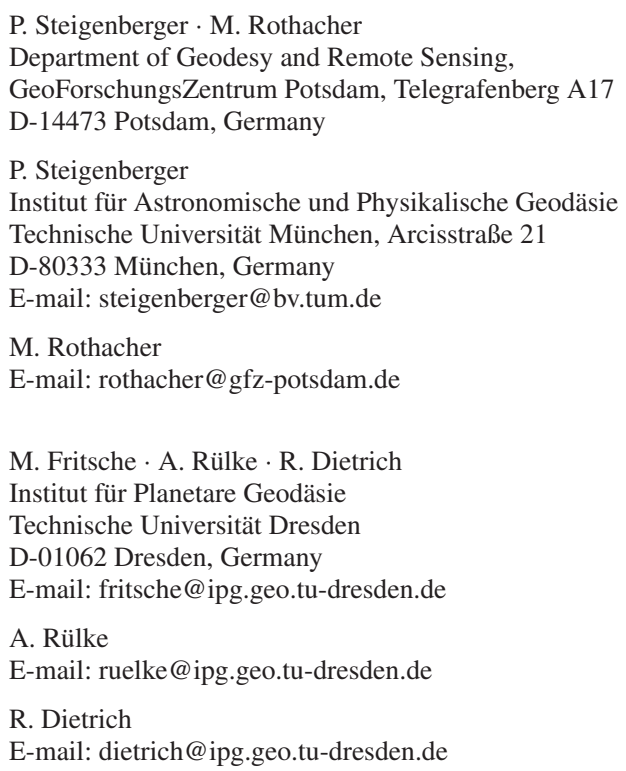




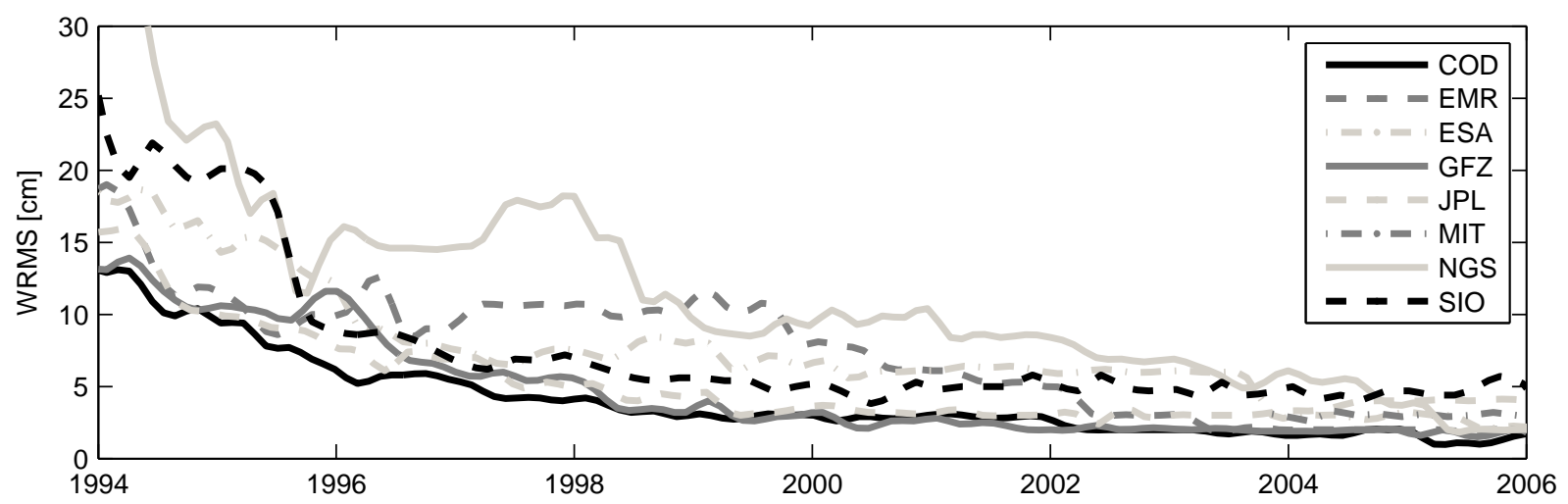

Fig. 1 Weighted RMS of the individual IGS analysis center solutions w.r.t. the IGS final orbits as given in the weekly orbit combination summary files (available at ftp://cddis.gsfc.nasa.gov/pub/gps/products).

of the orbits computed in the first years of the IGS with less sophisticated methods than nowadays could be significantly improved by reprocessing the raw GPS observation data with up-to-date models and processing strategies.

Therefore, Technische Universität München (TUM), Technische Universität Dresden (TUD) and GeoForschungsZentrum (GFZ) Potsdam reprocessed a global GPS tracking network of 202 stations starting with 1 January 1994 in a joint effort. The Bernese GPS Software (Dach et al 2007) and a processing scheme similar to that of the IGS AC at the Center for Orbit Determination in Europe (CODE) in Bern (Hugentobler et al 2005) have been used. First results of this reprocessing effort as regards station coordinates, Earth rotation and troposphere parameters are given in Steigenberger et al (2006), Vey et al (2006) and Steigenberger et al (2007). The quality and consistency of the orbits estimated within the TUM/TUD/GFZ reprocessing effort will be discussed in this paper.

Sect. 2 describes the GPS reprocessing and in particular the orbit modeling. The formal accuracy and the internal consistency of the reprocessed orbits as well as the improvement compared to the operational CODE orbits are evaluated in Sect. 3. Sect. 4 compares the reprocessed orbits with the IGS final orbits and an independent validation with Satellite Laser Ranging (SLR) is given in Sect. 5.

\section{GPS Reprocessing}

\subsection{Processing Strategy}

The general reprocessing strategy is described in detail in Steigenberger et al (2006) but the results of a more recent reprocessing run are discussed in this paper. Therefore, only the differences to this general strategy are described here. GPS observations of a global tracking network (202 stations) were completely and homogeneously reprocessed for the time interval 1 January 1994 till 31 October 2005. Major changes in the modeling w.r.t. Steigenberger et al (2006) are:

- Hydrostatic Isobaric Mapping Function (IMF, Niell 2000) computed from gridded ECMWF (European Centre for Medium-Range Weather Forecasts) z200 data provided by TU Vienna ${ }^{1}$ for the mapping of the hydrostatic troposphere a priori delays.

- Second and third order ionospheric corrections applied according to Fritsche et al (2005).

- TUM05 satellite antenna phase center variations and offsets. TUM05 is the contribution of TUM to the IGS absolute antenna phase center model igs05 (Schmid et al 2007).

The preparation, setup and realization of this reprocessing required about 7.5 man-years as well as about 20,000 hours of CPU time on LINUX clusters available at TUM and TUD. Such a reprocessing should be repeated whenever major model changes (like the switch from a relative to an absolute antenna phase center model in November 2006) are implemented within the IGS or a new version of the International Terrestrial Reference Frame (ITRF, e.g., Altamimi et al 2007) is computed.

\subsection{Orbit Modeling}

For the GPS satellites, orbiting the Earth at an altitude of about 20,200 km, the solar radiation pressure is the largest uncertainty in the orbit determination process. To account for this effect usually dynamical parameters are estimated in addition

\footnotetext{
1 http://mars.hg.tuwien.ac.at/ ecmwf1/
} 


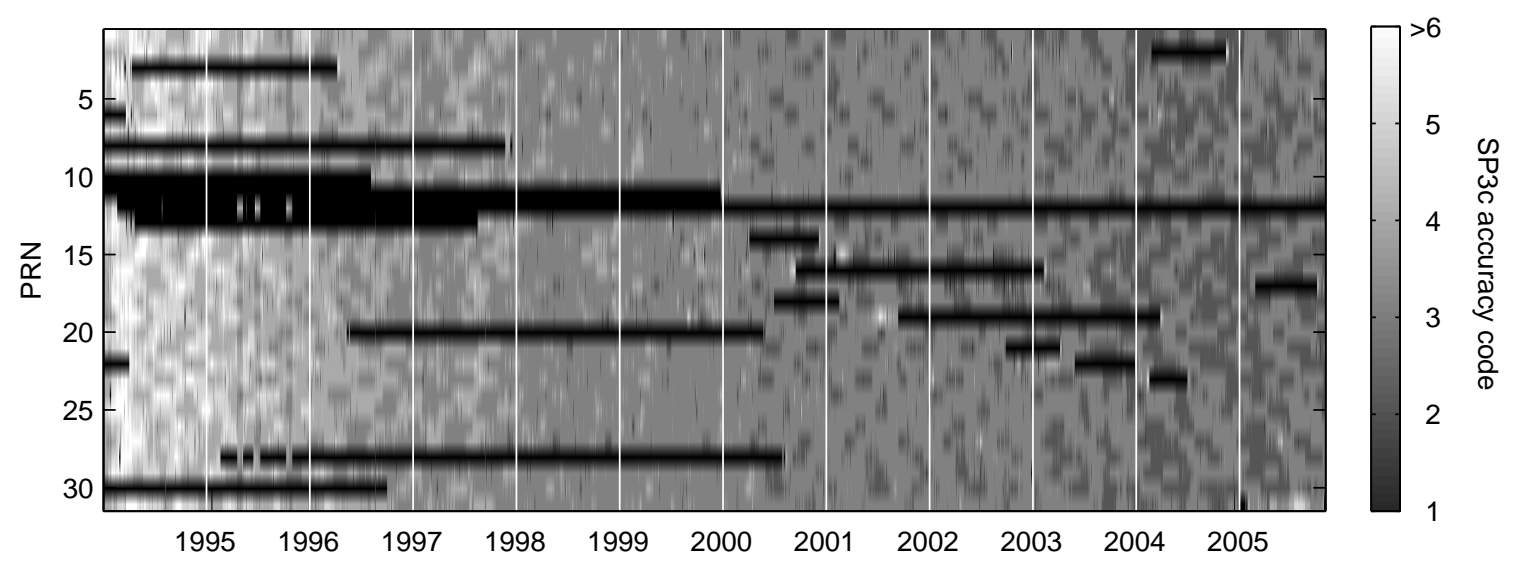

Fig. 2 SP3 accuracy codes of the 1-day orbits. Periods where a PRN was either unoccupied or excluded from the reprocessing are given in black.

to the six Keplerian elements. The Enhanced CODE orbit model described by Beutler et al (1994) has nine dynamical parameters:

$\mathbf{a}_{R P R}=v \cdot\left[\mathbf{a}_{a p r}+D(u) \cdot \mathbf{e}_{D}+Y(u) \cdot \mathbf{e}_{Y}+X(u) \cdot \mathbf{e}_{X}\right]$

with

$D(u)=D_{0}+D_{C} \cdot \cos u+D_{S} \cdot \sin u$

$Y(u)=Y_{0}+Y_{C} \cdot \cos u+Y_{S} \cdot \sin u$

$X(u)=X_{0}+X_{C} \cdot \cos u+X_{S} \cdot \sin u$

and

$\mathbf{a}_{R P R} \quad$ acceleration due to solar radiation pressure

$v \quad$ eclipse factor ( 1 in sunlight, 0 in the shadow)

$\mathbf{a}_{a p r} \quad$ a priori acceleration

$\mathbf{e}_{D} \quad$ unit vector pointing to the Sun

$\mathbf{e}_{Y} \quad$ unit vector in the direction of the solar panel axis

$\mathbf{e}_{X} \quad \mathbf{e}_{D} \times \mathbf{e}_{Y}$

$u \quad$ argument of latitude.

The three coefficients with index $0\left(D_{0}, Y_{0}, X_{0}\right)$ represent constant terms whereas the coefficients with indices $C$ and $S$ describe periodic variations in the three directions. Together with the six Keplerian elements, altogether 15 deterministic parameters can be used to characterize an orbital arc. However, only five of the nine radiation pressure parameters are estimated freely in our reprocessed solution: the three constant parameters and the periodic parameters in $X$-direction. The other parameters are heavily constrained to zero. The T10 and T20 implementations of the ROCK4 and ROCK42 models described by Fliegel et al (1992) have been used as a priori radiation pressure model for the Block I and II/IIA satellites and the model T30 (Fliegel and Gallini 1996) for the Block IIR satellites.

Pseudo-stochastic pulses (small velocity changes) in radial, along-track and cross-track direction are set up at 12-hour intervals. These pulses are constrained to zero with $10^{-6}, 10^{-5}$ and $10^{-8} \mathrm{~m} / \mathrm{s}^{2}$, respectively. The absence of the radiation pressure of the Sun when the satellite passes the shadow of the Earth is considered with a cylindric model, for the Moon an umbra/penumbra model is used. For orbit integration the JGM3 gravity field (Tapley et al 1996) up to degree and order 12 including secular variations for $J_{2}$ and the ocean tide model CSR 3.0 (Eanes and Bettadpur 1996) are applied. Solid Earth tides are accounted for according to the IERS conventions 2003 (McCarthy and Petit 2004). The effect of the pole tide is computed with constant pole offsets $\left(\bar{x}_{p}=0.033\right.$ as, $\bar{y}_{p}=0.331$ as) whereas the oceanic pole tide is neglected. The JPL ephemeris DE405 (Standish 1998) is used to derive the positions of the Moon and the Sun for computing third-body forces. The positions of the planets Venus, Mars and Jupiter are computed according to Seidelmann (1992) and their third-body forces are also considered. The general relativistic effect for the equation of motion (McCarthy and Petit 2004) is applied but geodesic and Lense-Thirring precession are neglected as these effects have no significant influence on GPS orbits.

Whereas the orbits discussed in Sect. 3 are based on 1-day solutions, the orbits discussed in Sect. 4 and 5 stem from the middle day of a 3-day solution where the station coordinates and Earth rotation parameters are fixed to the results of the weekly solution. These 3-day orbit arcs are usually represented by one set of osculating elements, radiation pressure parameters and pseudo-stochastic pulses at 12-hour intervals, thus strengthening the stability of the arcs. If this is not possible due to modeling problems or the maneuver of a particular satellite, a 3-day arc is split up at the day boundaries into two or even three arcs. 

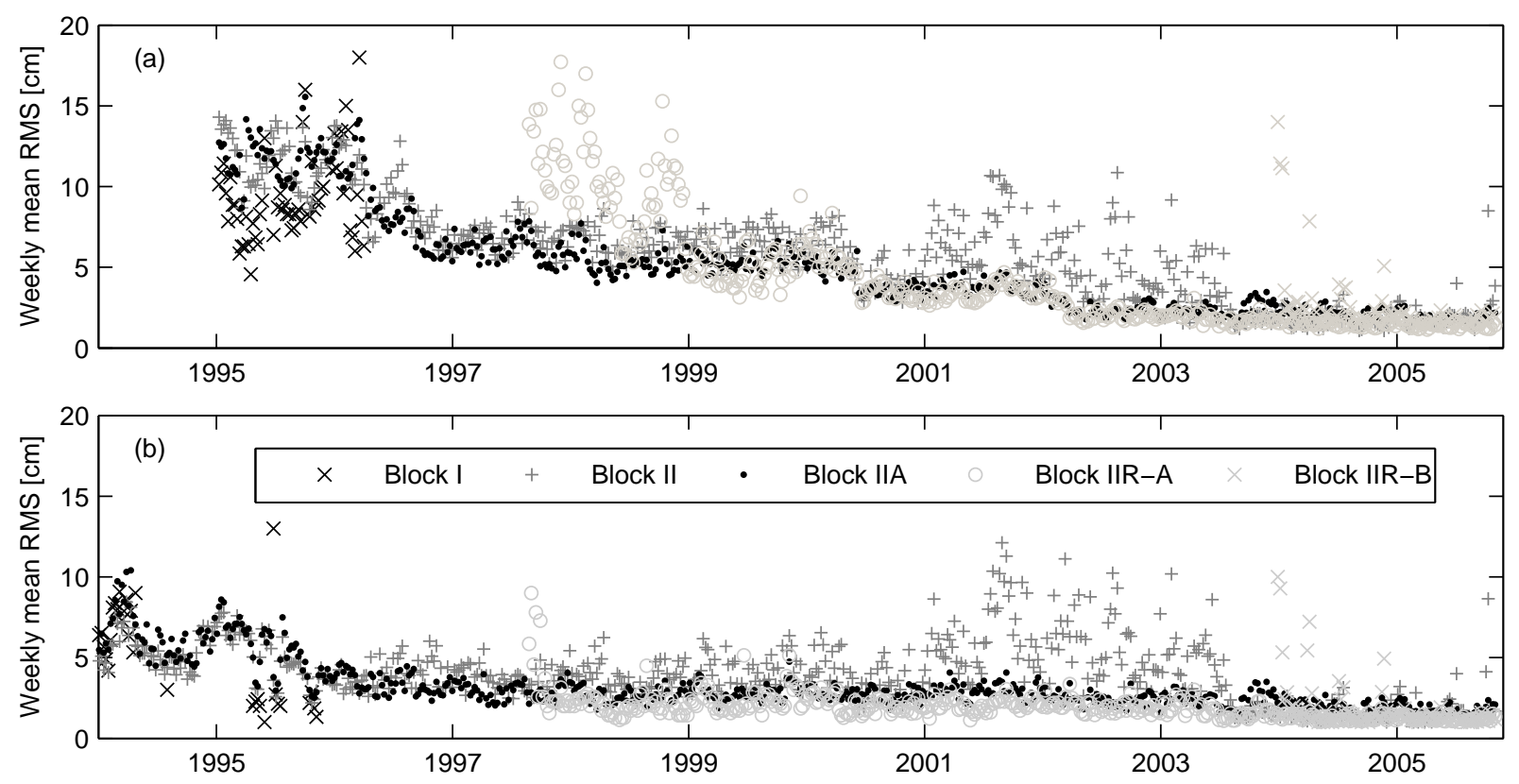

Fig. 3 Mean weekly RMS values of 3-day orbit fits through 1-day orbits for the different types of GPS satellites: (a) CODE final orbits (RMS values only available starting with GPS week 782); (b) reprocessed orbits.

\section{Formal Accuracy and Internal Consistency}

An indicator for the formal accuracy of satellite orbits is given in the header of the SP3 $\mathrm{c}^{2}$ orbit files. This so-called SP3c accuracy code $n$ allows for a weighting of the individual satellites and stands for a formal position accuracy of $\pm 2^{n}$ millimeters. The SP3c accuracy codes of the reprocessed 1-day orbits (derived from the formal errors of the argument of latitude) are shown in Fig. 2. In 1994 and 1995 the accuracy is worse, a fact that is also visible in the internal consistency tests that will be discussed below. For most satellites, the accuracy codes are worse by a factor of $2-3$ during the very first weeks after the launch. A limiting factor for the accuracy of the orbit determination immediately after the launch is the small number of tracking stations recording observations of new satellites. E.g., only receivers with an all-in-view option are able to track satellites that are set unhealthy in the almanac as it is done for all satellites before the official commissioning. But even if a satellite is set healthy, it usually takes several days until all receivers actually track this satellite. Another effect degrading the orbit accuracy during the first weeks after the launch are unmodeled accelerations due to outgasing (Springer 2000).

To quantify the internal consistency of the satellite orbits, 3-day orbit arcs were fitted through 3 consecutive 1-day orbits. The mean RMS of the 3-day arc w.r.t. the three 1-day arcs serves as quality indicator. Weekly means of these RMS values for the different satellite blocks are shown in Fig. 3. The increased RMS values for the Block II satellites between 2001 and 2003 that are present in both, the CODE and the reprocessed series are related to the aging of some of these satellites (problems with momentum wheels resulting in thruster firing for attitude control). The situation improves in mid of 2003 when the attitude control system of two of these problematic Block II satellites (SVN 15 and 17) was changed. In 1995 the RMS values could be reduced by a factor of about two by reprocessing the orbits. During three short time periods in 1995, the RMS values of the reprocessed orbits are smaller by a factor of about two due to the temporary deactivation of anti-spoofing (see Tab. 3 in Steigenberger et al (2006)). Between 1996 and 2000, the RMS values of the CODE orbits are in general above the $5 \mathrm{~cm}$ level whereas the reprocessed orbits are on a level below $5 \mathrm{~cm}$. A clear improvement of the CODE orbits can be seen in June 2000 when the pseudo-stochastic pulses were changed. After switching to an improved ambiguity resolution strategy in March 2002 (Hugentobler et al 2004), the internal consistency of the CODE and the reprocessed orbits is on almost the same level.

However, even in 2004 the reprocessed orbits show in general slightly smaller RMS values than the CODE orbits although the processing strategy of both solutions is very similar. This effect is attributed to the application of an absolute antenna phase center model for receiver and satellite antennas for the reprocessed solution (CODE used the relative model until 4 November 2006 like the other IGS ACs) resulting in a higher internal consistency of the orbits, see Schmid et al (2007).

Besides the general improvement in the early years, the improvement for the Block IIR-A satellites (actually only one satellite for more than two years) is striking. SVN 43 (PRN 13) was the first Block IIR-A satellite in orbit and the vertical antenna offset was unknown. Therefore, the offset of the Block II/IIA satellites $(1.023 \mathrm{~m})$ was used by the IGS ACs for this

\footnotetext{
${ }^{2}$ Format description available at http://igscb.jpl.nasa.gov/igscb/data/format/sp3c.txt
} 


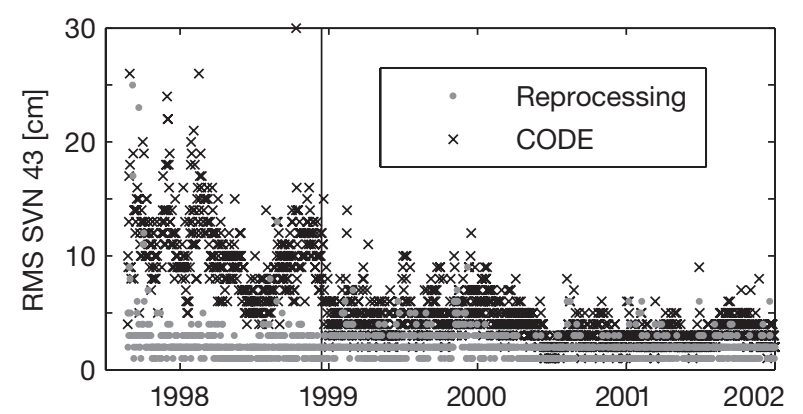

Fig. 4 RMS values of 3-day orbit fits through 1-day orbits for SVN 43 (PRN 13). The change of the $z$-offset used by the CODE AC is indicated by a vertical line.

Block IIR-A satellite, too. This assumption led to large and varying RMS values for the orbit fits (see Fig. 4) indicating that this offset was not appropriate. Offset estimations by CODE, GFZ and Jet Propulsion Laboratory (JPL) showed that the $z$-offset seemed to differ from the value of the Block II/IIA satellites. Therefore, a $z$-offset value of $0.000 \mathrm{~m}$ was adopted by the IGS on 29 November 1998 (Kouba and Mireault 1999). After changing the offset, the orbit quality was significantly improved and also the large variations disappeared. In the reprocessed time series, the RMS values for SVN 43 are almost constant for the whole time period. The slightly larger values at the beginning are probably due to the fact that shortly after the launch the satellite was tracked by a small number of stations only.

\section{Comparisons with IGS Orbits}

The reprocessed orbits have been compared to the IGS final orbits by 7-parameter similarity transformations. Several reference frame changes present in the IGS orbits can clearly be seen in the transformation parameters. The translations dX, $\mathrm{dY}$ and $\mathrm{dZ}$, the rotations $\mathrm{rX}, \mathrm{rY}, \mathrm{rZ}$ as well as the scale difference $\mathrm{dS}$ are shown in Fig. 5. The largest rotations as well as rotation rates show up for ITRF92 and ITRF93. Whereas for ITRF93 till ITRF2000 (in fact the IGS implementations of ITRF2000, namely IGS00 and IGb00, Ray et al 2004) the NNR-NUVEL1A model (DeMets et al 1994) was used for the realization of the no-net-rotation (NNR) datum, NNR-NUVEL1 (Argus and Gordon 1991) was used for ITRF92 (Boucher et al 1993). ITRF93 is slightly rotated w.r.t. NNR-NUVEL1A as the time evolution of the Earth rotation parameters (ERPs) estimated in the solutions contributing to ITRF93 does not follow the NNR-NUVEL1A model (Boucher et al 1994). For the translations, no clear discontinuities are visible although large drifts in dY are obvious for ITRF92 and ITRF93. The absence of translation discontinuities has two different reasons: (1) For ITRF94, ITRF96 and ITRF97 a continuity condition for the origin has been applied (McCarthy and Petit 2004). (2) The impact of a shift in the reference frame on the center of mass determined by the GPS orbits is smaller by a factor of 5-10 than the shift itself (Ferland et al 2005). The scale offset does not significantly differ for ITRF92 till ITRF97 but is larger by almost a factor of two for ITRF2000. In addition, the scatter of the scale gets significantly smaller in mid of 2003. The reason for this effect is unknown. The general scale offset is probably related to the different models and software packages used by the IGS ACs.

The mean RMS of the transformed reprocessed orbits w.r.t. the IGS final orbits is shown in Fig. 6. In the first year, the RMS is on a level of $10-15 \mathrm{~cm}$ and falls below $10 \mathrm{~cm}$ in mid of 1995. In the following years, the RMS steadily decreases, reaches a level of about $2 \mathrm{~cm}$ in 2003 and is then on the same level as the RMS of those IGS ACs with the smallest RMS values (Gendt and Kouba 2008).

\section{Validation with Satellite Laser Ranging}

As two of the GPS satellites (SVN 35 and 36) are equipped with laser retroreflector arrays (LRAs), these satellites can be tracked with SLR. Thus, the satellite orbits computed from the GPS microwave observations (basically the radial component due to the large orbit height of the GPS satellites) can be validated by an independent (optical) technique. A general introduction to the SLR tracking of GPS satellites is given in Degnan and Pavlis (1994), recent results can be found in Appleby and Otsubo (2005) and Urschl et al (2005, 2007, 2008).

In the following, range residuals, i.e. differences between the measured SLR range and the range computed from the GPS-derived orbits, will be used for comparisons (i.e., no parameters are estimated). For the computation of these laser residuals, the coordinates of the SLR stations have been fixed to the rescaled version of ITRF2005 ${ }^{3}$ (Altamimi et al 2007). This version is consistent with the scale determined by SLR as the scale of the original version of ITRF2005 (determined by

\footnotetext{
${ }^{3}$ http://itrf.ensg.ign.fr/ITRF_solutions/2005/ITRF2005_SLR.php
} 

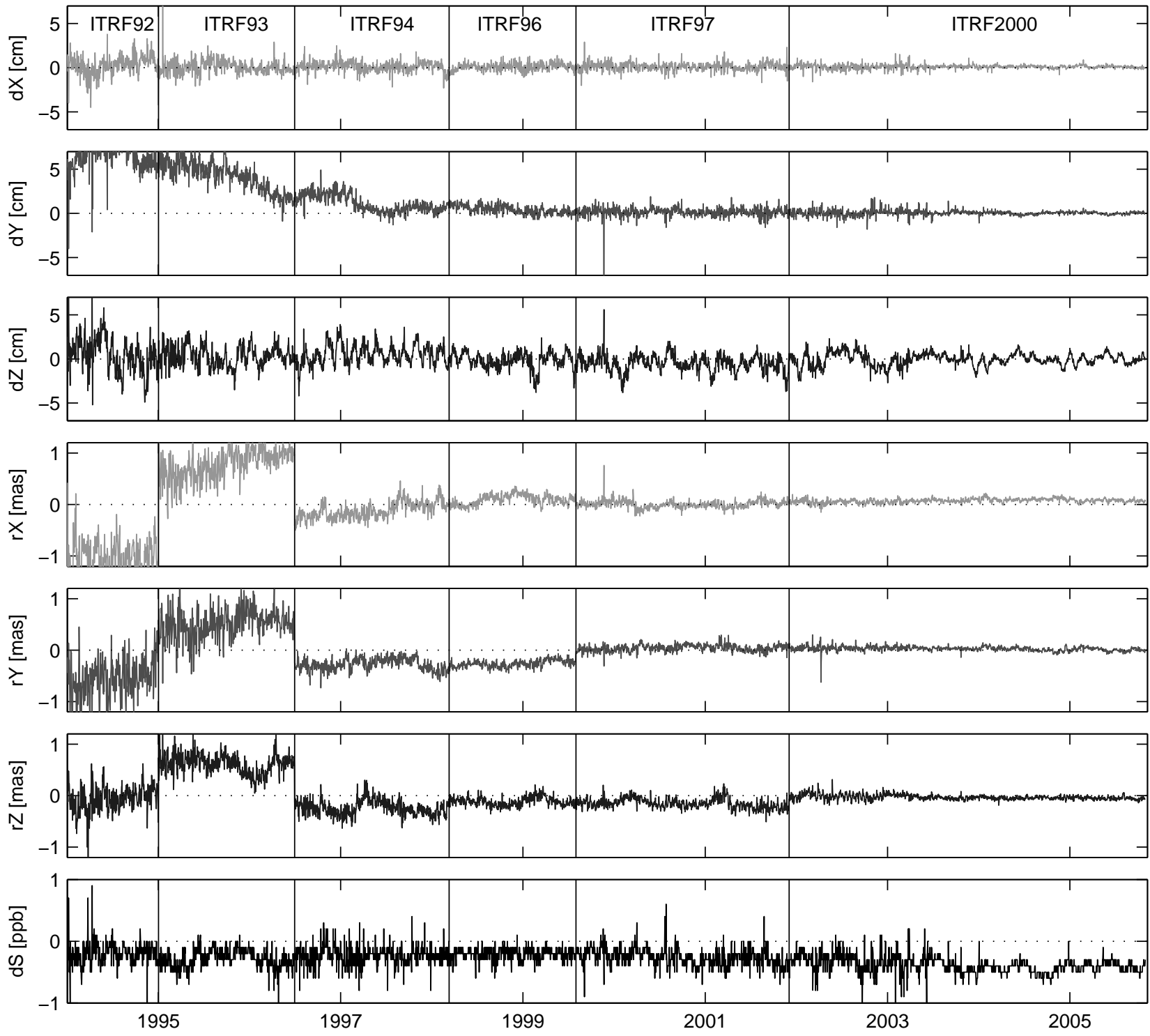

Fig. 5 Transformation parameters of daily 7-parameter similarity transformations between the IGS and the reprocessed orbits: translations dX, dY, $\mathrm{dZ}$; rotations $\mathrm{rX}, \mathrm{rY}, \mathrm{rZ}$ and scale $\mathrm{dS}$.

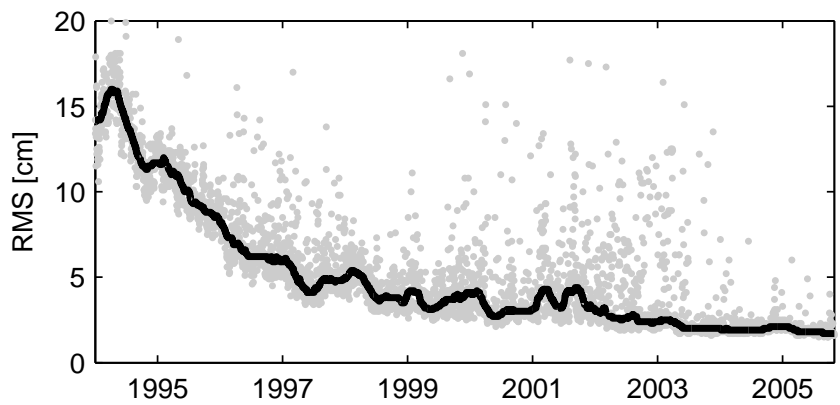

Fig. 6 Mean RMS of transformed reprocessed orbits w.r.t. the IGS final orbits. The solid line represents a 100-day median. 
Table 1 SLR range residuals for GPS-derived satellite orbits of SVN 35 and 36 determined by the IGS, CODE and the reprocessing: mean bias, standard deviation (STD) and approximate number of normal points (\# NPs).

\begin{tabular}{lllrrr}
\hline \multirow{2}{*}{ SVN } & Time int. & \multicolumn{2}{c}{$01 / 94-10 / 05$} & \multicolumn{2}{c}{$01 / 98-10 / 05$} \\
\cline { 2 - 6 } & & $\begin{array}{r}\text { Bias } \\
{[\mathrm{mm}]}\end{array}$ & $\begin{array}{r}\text { STD } \\
{[\mathrm{mm}]}\end{array}$ & $\begin{array}{r}\text { Bias } \\
{[\mathrm{mm}]}\end{array}$ & $\begin{array}{r}\text { STD } \\
{[\mathrm{mm}]}\end{array}$ \\
\hline \multirow{2}{*}{35} & \# NPs & \multicolumn{2}{c}{35,800} & \multicolumn{2}{c}{22,800} \\
& IGS & -41.8 & 35.7 & -37.0 & 21.8 \\
& CODE & -50.6 & 38.1 & -45.1 & 24.0 \\
\multirow{3}{*}{36} & Reprocessing & -46.2 & 27.6 & -46.0 & 21.0 \\
& \# NPs & 28,900 & 20,100 \\
& IGS & -35.4 & 35.2 & -34.8 & 26.7 \\
& CODE & -51.0 & 38.4 & -49.1 & 29.0 \\
& Reprocessing & -53.1 & 30.9 & -52.8 & 26.2 \\
\hline
\end{tabular}

VLBI) clearly differs from the SLR scale. The reflector offsets provided by the International Laser Ranging Service ${ }^{4}$ have been applied. The model of Marini (1972) together with the meteorological observations at the SLR sites have been used to correct for the tropospheric delay of the SLR observations. As the Bernese GPS software is also used for the computation of the SLR residuals, full consistency of the models applied (e.g., solid Earth tides and ocean loading) is guaranteed.

More than 67,000 SLR normal points (NPs) during the time period January 1994 till October 2005 were used to compute the residuals of the microwave-derived GPS orbits. The number of normal points is smaller for SVN 36 as this satellite was only launched in March 1994. The laser tracking of SVN 36 started in April 1994 (SVN 35 laser tracking started in October 1993) and had a lower tracking priority compared to SVN 35. Outliers exceeding an absolute value of $0.5 \mathrm{~m}$ or the 5 -fold mean standard deviation (STD) of each station have been excluded. $1.7 \%$ and $1.4 \%$ of the normal points met these criteria for the reprocessed orbits of SVN 35 and 36, respectively.

Mean bias and standard deviation of the SLR residuals for CODE, IGS and the reprocessed orbits are given in Tab. 1. Springer (2000) showed that the residuals during the eclipse phase are significantly increased due to deviations from nominal attitude and the comparatively big distance of more than $1 \mathrm{~m}$ between the rotation axis and the LRA. Therefore, normal points during eclipses have been excluded from Tab. 1. As the scatter of the IGS residual time series is almost on the same level as the scatter of the reprocessed orbit residuals after 1997 (not shown here), bias and standard deviation for a limited time interval covering January 1998 till October 2005 are also included in Tab. 1. By reprocessing the GPS orbits, the mean standard deviation for the full time interval could be reduced by about $1 \mathrm{~cm}$ compared to the CODE orbits and by $8 \mathrm{~mm}$ (SVN 35) and $4 \mathrm{~mm}$ (SVN 36) compared to the IGS orbits. The standard deviations of the reduced time interval are almost identical for the IGS and the reprocessed orbits and only a few millimeters larger for the CODE orbits.
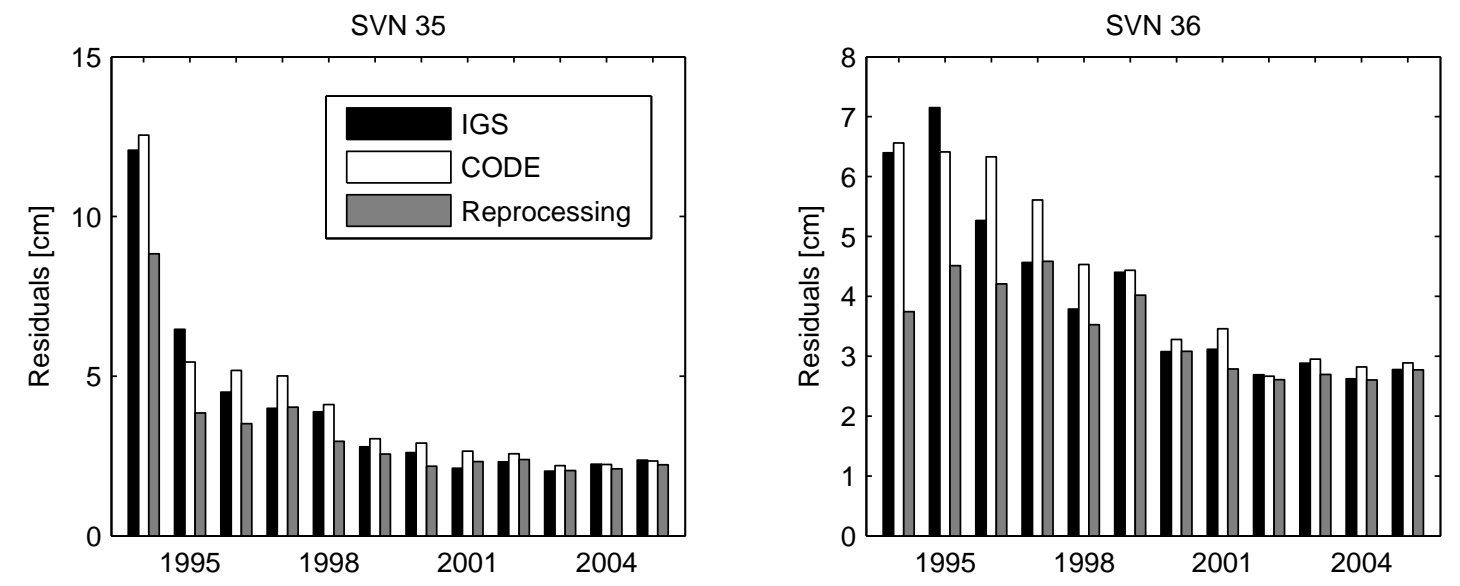

Fig. 7 Yearly mean SLR residuals for IGS, CODE and reprocessed orbits of SVN 35 and SVN 36. Note the different scale of the $y$-axis.

To get a more detailed impression of the STD improvement with time, Fig. 7 shows yearly mean standard deviations for SVN 35 and 36. In 1994 and 1995 the mean STD of the reprocessed orbits is 30-40\% smaller compared to the IGS and CODE orbits. The increased STD values in 1994 of SVN 35 compared to SVN 36 can be explained by in particular large

\footnotetext{
4 http://ilrs.gsfc.nasa.gov/satellite_missions/center_of_mass/index.html
} 
residuals of up to $50 \mathrm{~cm}$ at the beginning of 1994 (before the launch of SVN 36 in March 1994). In the following years, the STD differences get smaller, in particular for the IGS orbits compared to the reprocessed orbits. Starting with 2002, the STD of all three orbit solutions is on almost the same level. These results indicate that a significant improvement in the orbit quality can be achieved by completely reprocessing the orbits in the years $1994-1997$. On the other hand, the orbit quality - as evaluated by SLR - can only slightly be improved after 2000 . However, one has to be aware that the laser residuals do not only reflect the GPS orbit accuracy but also errors in the laser ranging technology. Therefore, the improvements during the early years as described above might be too pessimistic as the SLR technology has also been improved during the time period considered.

Due to the increased scatter of the IGS and CODE orbit residuals in the first years, only the limited time interval will be considered for the comparison of the biases. However, it should be mentioned that the biases of the reprocessed orbits differ only by a few tenth of a millimeter for the full and the limited time interval for both, SVN 35 and 36 . The wellknown systematic bias of a few centimeters (e.g., Urschl et al 2008) between the optical technique SLR and the microwave technique GPS can also be seen in the reprocessed orbits. As the sign of this bias is negative, the distance measured by SLR is shorter than the GPS-derived distance. The true origin of this bias is still unknown, Urschl et al (2007) assumed that deficiencies in the dynamic model of the GPS orbits might be the reason for the bias. Whereas the biases of the CODE and the reprocessed orbits differ only by a few millimeters, the bias of the IGS orbits is smaller by about $1 \mathrm{~cm}$ for SVN 35 and almost $2 \mathrm{~cm}$ for SVN 36. This effect can be explained by the different orbit scales of the IGS ACs differing by up to $\pm 0.5 \mathrm{ppb}$ from the combined IGS orbit scale (Gendt and Kouba 2008).

\section{Conclusions}

High-precision GPS satellite orbits provide the basis for a number of geodetic studies like monitoring of deformations, postglacial rebound or sea level rise. Long-term studies based on operational (and therefore inhomogeneous and inconsistent) GPS orbits suffer from the degraded quality and consistency of these orbits, in particular in the first years after the establishment of the IGS in 1994. To overcome these problems, TUM, TUD and GFZ performed a complete reprocessing of a global GPS network starting in 1994 including the estimation of satellite orbits. The orbits also provide a valuable basis for homogeneous reanalyses of regional networks (e.g., Völksen 2008).

In particular from 1994 till 1997, the internal consistency (evaluated by orbit fits) as well as the external quality evaluated by SLR could be significantly improved by homogeneously reprocessing the GPS satellite orbits with an up-to-date processing strategy. However, the orbit quality in these early years is still worse compared to nowadays due the smaller number of stations and less sophisticated receivers. At present, the reprocessed orbits are on the same quality level as the IGS AC submissions. A further improvement could be achieved by combining reprocessed orbits computed by different ACs. Therefore, a reprocessing effort was initiated by the IGS (Steigenberger et al 2008) and has already started with a test phase of three months. A similar reprocessing of GLONASS observations is expected to result in comparable improvements. However, such a GLONASS reprocessing or even a combined GPS/GLONASS reprocessing remains a challenging task for the future.

The GPS satellite orbits discussed in this paper (SP3c and internal Bernese format) and consistent ERPs are publicly available at the Information Systems and Data Center ${ }^{5}$ of GFZ Potsdam or at TU Dresden ${ }^{6}$.

Acknowledgements The GPS reprocessing project was funded by Deutsche Forschungsgemeinschaft (German Research Foundation), project number 233069. The authors would like to thank the CODE IGS AC team for their support to modify the processing scheme and the Bernese GPS software, Johannes Boehm for providing ECMWF z200 data for IMF, Urs Hugentobler for valuable discussions, the International GNSS Service (Dow et al 2005) for providing GPS observation data and the International Laser Ranging Service (Pearlman et al 2002) for providing SLR observation data. The constructive comments of Jim Ray and an anonymous reviewer helped to improve the quality of this article.

\section{References}

Altamimi Z, Collilieux X, Legrand J, Garayt B, Boucher C (2007) ITRF2005: A new release of the International Terrestrial Reference Frame based on time series of station positions and Earth Orientation Parameters. J Geophys Res 112:B09401, DOI 10.1029/2007JB004949

Appleby GM, Otsubo T (2005) Laser Ranging as a Precise Tool to Evaluate GNSS orbital solutions. In: Garate J, Davila J, Noll C, Pearlman M (eds) 14th International Workshop on Laser Ranging, Real Instituto y Observatorio de la Armada, San Fernando, Boletin ROA, vol 5/2005

Argus D, Gordon R (1991) No-net-rotation model of current plate velocities incorporating plate motion model NUVEL-1. Geophys Res Let 111(18):2039-2042

Beutler G (1993) The 1992 IGS Test Campaign, Epoch'92, and the IGS Pilot Service: An overview. In: Beutler G, Brockmann E (eds) Proceedings of the 1993 IGS Workshop, Druckerei der Universität Bern, Berne, pp 3-9

Beutler G, Brockmann E, Gurtner W, Hugentobler U, Mervart L, Rothacher M (1994) Extended orbit modeling techniques at the CODE Processing Center of the International GPS Service (IGS): Theory and initial results. Manuscr Geod 19:367-386

\footnotetext{
5 http://isdc.gfz-potsdam.de/gps-pdr

6 http://tpg.geo.tu-dresden.de/ipg/forschung/projekte/gpsrepro.htm
} 
Boucher C, Altamimi Z, Duhem L (1993) ITRF 92 and its associated velocity field. IERS Tech. Note 15, Observatoire de Paris, Paris

Boucher C, Altamimi Z, Duhem L (1994) Results and analysis of the ITRF93. IERS Tech. Note 18, Observatoire de Paris, Paris

Dach R, Hugentobler U, Fridez P, Meindl M (eds) (2007) Bernese GPS Software Version 5.0. Astronomical Institute, University of Bern, Bern, Switzerland

Degnan JJ, Pavlis EC (1994) Laser ranging to GPS satellites with centimeter accuracy. GPS World 5(9):62-70

DeMets C, Gordon R, Argus D, Stein S (1994) Effect of recent revisions to the geomagnetic reversal timescale on estimates of current plate motions. Geophys Res Lett 21(20):2191-2194

Dow J, Neilan R, Gendt G (2005) The International GPS Service: Celebrating the 10th anniversary and looking to the next decade. Adv Space Res 36(3):320-326, DOI 10.1016/j.asr.2005.05.125

Eanes R, Bettadpur S (1996) The CSR 3.0 global ocean tide model. Technical Memorandum CSR-TM-96-05, Center for Space Research, Austin, TX, USA

Ferland R, Gendt G, Schöne T (2005) IGS reference frame maintenance. In: Meindl M (ed) Celebrating a Decade of the International GPS Service, Workshop and Symposium 2004, Astronomical Institute, University of Berne, Berne, Switzerland

Fliegel H, Gallini T, Swift E (1992) Global Positioning System radiation force model for geodetic applications. J Geophys Res 97(B1):559-568

Fliegel HF, Gallini TE (1996) Solar force modeling of Block IIR Global Positioning System satellites. J Spacecr Rockets 33(6):863-866

Fritsche M, Dietrich R, Knöfel C, Rülke A, Vey S, Rothacher M, Steigenberger P (2005) Impact of higher-order ionospheric terms on GPS estimates. Geophys Res Lett 32:L23311, DOI 10.1029/2005GL024342

Gendt G, Kouba J (2008) Quality and consistency of the IGS combined products. In: IGS Workshop 2006 Proceedings, ESA, Darmstadt

Hugentobler U, Schaer S, Beutler G, Bock H, Dach R, Jäggi A, Meindl M, Urschl C, Mervart L, Rothacher M, Wild U, Wiget A, Brockmann E, Ineichen D, Weber G, Habrich H, Boucher C (2004) CODE IGS analysis center technical report 2002. In: Gowey K, Neilan R, Moore A (eds) International GPS Service 2001-2002 Technical Report, IGS Central Bureau, Jet Propulsion Laboratory, Pasadena

Hugentobler U, Schaer S, Dach R, Meindl M, Urschl C (2005) Routine processing of combined solutions for GPS and GLONASS at CODE. In: Meindl M (ed) Celebrating a Decade of the International GPS Service, Workshop and Symposium 2004, Astronomical Institute, University of Berne, Berne, Switzerland, published on CD-ROM

Kouba J, Mireault Y (1999) 1998 Analysis Coordinator report. In: Gowey K, Neilan R, Moore A (eds) International GPS Service for Geodynamics 1998 Technical Reports, IGS Central Bureau, Jet Propulsion Laboratory, Pasadena

Marini J (1972) Correction of satellite tracking data for an arbitrary tropospheric profile. Radio Sci (7):223-231

McCarthy D, Petit G (2004) IERS Conventions (2003). IERS Tech. Note 32, Verlag des Bundesamtes für Kartographie und Geodäsie, Frankfurt

Niell A (2000) Improved atmospheric mapping functions for VLBI and GPS. Earth Planets Space 52(10):699-702

Pearlman MR, Degnan JJ, Bosworth JM (2002) The International Laser Ranging Service. Adv Space Res 30(2):125-143

Ray J, Dong D, Altamimi Z (2004) IGS reference frames: status and future improvements. GPS Sol 8(4):251-266, DOI 10.1007/ s10291-004-0110-x

Schmid R, Steigenberger P, Gendt G, Ge M, Rothacher M (2007) Generation of a consistent absolute phase center correction model for GPS receiver and satellite antennas. J Geod 81(12):781-798, DOI 10.1007/s00190-007-0148-y

Seidelmann PK (1992) Explanatory supplement to the astronomical almanac. University Science Books, Mill Valley, California, ISBN: 0-93570268-7

Springer T (2000) Modelling and validating orbits and clocks using the Global Positioning System. Geodätisch Geophysikalische Arbeiten in der Schweiz, 60, Zürich, Switzerland

Standish E (1998) JPL planetary and lunar ephemerides, DE405/LE405. Interoffice Memorandum IOM 312.F-98-048, Jet Propulsion Laboratory, Pasadena

Steigenberger P, Rothacher M, Dietrich R, Fritsche M, Rülke A, Vey S (2006) Reprocessing of a global GPS network. J Geophys Res 111:B05402, DOI 10.1029/2005JB003747

Steigenberger P, Tesmer V, Krügel M, Thaller D, Schmid R, Vey S, Rothacher M (2007) Comparisons of homogeneously reprocessed GPS and VLBI long time-series of troposphere zenith delays and gradients. J Geod 81(6-8):503-514, DOI 10.1007/s00190-006-0124-y

Steigenberger P, Romero I, Fang P (2008) Reprocessing Issues, Standardization, New Models. In: IGS Workshop 2006 Proceedings, ESA, Darmstadt

Tapley B, Watkins M, Ries J, Davis G, Eanes R, Poole S, Rim H, Schutz B, Shum C, Nerem R, Lerch F, Marshall J, Klosko S, Pavlis N, Williamson R (1996) The Joint Gravity Model 3. J Geophys Res 101(B12):28,029-28,049, DOI 10.1029/96JB01645

Urschl C, Gurtner W, Hugentobler U, Schaer S, Beutler G (2005) Validation of GNSS orbits using SLR observations. Adv Space Res 36(3):412417, DOI 10.1016/j.asr.2005.03.021

Urschl C, Beutler G, Gurtner W, Hugentobler U, Schaer S (2007) Contribution of SLR tracking data to GNSS orbit determination. Adv Space Res 39(10):1515-1523, DOI 10.1016/j.asr.2007.01.038

Urschl C, Beutler G, Gurtner W, Hugentobler U, Schaer S (2008) Validation of GNSS orbits using SLR observations. In: IGS Workshop 2006 Proceedings, Darmstadt

Vey S, Dietrich R, Fritsche M, Rülke A, Rothacher M, Steigenberger P (2006) Influence of mapping function parameters on global GPS network analyses: Comparisons between NMF and IMF. Geophys Res Lett 33:L01814, DOI 10.1029/2005GL024361

Völksen C (2008) Reprocessing of a regional GPS network in EUROPE. In: Sideris M (ed) Observing our Changing Earth, Springer, Berlin, Heidelberg, International Association of Geodesy Symposia, vol 133, pp 57-64, DOI 10.1007/978-3-540-85426-5_7 